Research article

\title{
Characteristics of community acquired and hospital acquired methicillin resistant Staphylococcus aureus isolates in the National Hospital of Sri Lanka
}

\author{
WAMP Samaranayake ${ }^{1}$, L Karunanayake ${ }^{1}$, CGUA Patabendige ${ }^{2}$ \\ Sri Lankan Journal of Infectious Diseases 2019 Vol.9(1)24-31 \\ DOI: http://dx.doi.org/10.4038/sljid.v9i1.8229
}

\begin{abstract}
Introduction and Objectives: Highly virulent community acquired methicillin resistant Staphylococcus aureus (MRSA) strains emerged recently causing infections in healthy young adults without predisposing factors. This descriptive cross-sectional study was conducted to compare socio-demography of patients and microbiology and molecular characteristics of Community acquired (CA) and Hospital acquired (HA) methicillin resistant $S$. aureus strains isolated at the National Hospital of Sri Lanka.
\end{abstract}

Methods and Results: Antimicrobial susceptibility test and Panton Valentine Leukocidine (PVL) gene detection was carried out on 100 MRSA isolates. CDC epidemiological criteria were used for differentiation of CA and HA MRSA. Of those 100 isolates, $21(21 \%)$ were CA-MRSA and 79(79\%) were HA-MRSA. Patients did not show any significant difference in acquiring CA MRSA and HA MRSA in relation to their age, sex and gender except ethnicity. The majority of these isolates were from pus samples. CA-MRSA isolates were significantly more sensitive to ciprofloxacin, fusidic acid, tetracycline, cotrimoxazole, and gentamicin compared with HA-MRSA isolates $(\mathrm{p}<0.001)$. Inducible, constitutive clindamycin resistance $(p<0.001)$ and multidrug resistant phenotypes were significantly higher $(p<0.001)$ among patients with HA-MRSA infection. All isolates were susceptible to glycopeptides, rifampicin and linezolid. Mupirocin resistance was seen in $6 \%$ and all isolates came from patients who harboured HA-MRSA strains $(\mathrm{p}<0.338)$. The PVL gene $(\mathrm{P}<0.001)$ was present in $20(95.2 \%)$ of CA-MRSA isolates.

Conclusion: This study highlights the importance of accurate differentiation of CA and HA MRSA using epidemiological, microbiological and molecular characteristics. Further, awareness of the existence of these types will optimise individual treatment strategies.

Key words: CA-MRSA, HA-MRSA, Antimicrobial resistance, Sri Lanka

${ }^{1}$ Department of Bacteriology, Medical Research Institute, Colombo 08, Sri Lanka ${ }^{2}$ Department of Microbiology, National Hospital of Sri Lanka, Colombo, Sri Lanka Address for correspondence: Dr Manori Samaranayake, Unit 51,105 Bridge Road, Westmead, New South Wales, Australia Telephone: +61(02)0437208795

Email:manorisamaranayake1981@gmail.com (iD https://orcid.org/0000-0003-2459-9006

Received 25 September 2018 and revised version accepted 8 February 2019 


\section{Introduction}

Methicillin resistant Staphylococcus aureus (MRSA) is one of the major nosocomial pathogens in Sri Lanka that causes mild to life threatening infections. Prevalence of MRSA in Sri Lanka varies among the hospital settings from $47 \%$ to $62 \%$ while most are resistant to many antimicrobials tested. ${ }^{1,2,3}$ In the late $1990 \mathrm{~s}$, a phenotypically and genotypically distinct highly virulent MRSA clone emerged as communityacquired/associated MRSA (CA-MRSA) causing skin and soft tissue infection, and severe haemorrhagic pneumonia in children and young adults without any predisposing conditions. ${ }^{4}$ It usually carries smaller staphylococcal cassette chromosome mec (SCCmec) elements e.g. IV, V that do not contain other resistance genes and many clones spread independently worldwide. They produce PantonValentine Leukocidin toxin (PVL) which is responsible for both skin infection and severe haemorrhagic necrotizing pneumonia through tissue necrosis and abscess formation. ${ }^{5}$ Many studies have shown significant association of PVL gene with CAMRSA isolates compared with HA-MRSA isolates despite its controversial significance. ${ }^{5,6}$ However, the prevalence of CA-MRSA varies markedly worldwide. Song et al. showed $25.5 \%$ prevalence of CA-MRSA in Asian countries with Sri Lanka demonstrating a higher prevalence of $38.8 \%$ in a multicentre surveillance study. ${ }^{7}$ Local studies to assess the burden and characteristics of CA-MRSA and HAMRSA infections in the country are lacking.

The purpose of this study was to compare the presence of PVL gene and antimicrobial susceptibility of CA and HA MRSA strains and to compare the socio-demographic features in patients with CA-MRSA and HA-MRSA infections in the National Hospital of Sri Lanka (NHSL).

\section{Method}

A descriptive cross-sectional study was conducted from November 2013 to March 2014 for statistically calculated 100 consecutive, non-repetitive MRSA isolates collected from the microbiology laboratory, National Hospital of Sri Lanka (NHSL).

HA-MRSA infection was defined as isolation of MRSA in a patient 48 hours after admission, with a history of hospitalization, surgery, dialysis, or residence in a longterm health care facility within the last one year prior to the culture date or who had an indwelling intravenous line, catheter or any other percutaneous medical device present at the time of isolation. Isolates with none of the above were classified as CAMRSA. ${ }^{8}$ An interviewer administered pre-piloted questionnaire was filled after written informed consent. Bed head tickets, clinic records and the guardian's histories were used in addition.

MRSA isolates were identified using standard protocols ${ }^{9,10}$ in the microbiology laboratory, Medical Research Institute (MRI). Methicillin resistance was screened with cefoxitin $30 \mu \mathrm{g}$ disk and confirmed by PBP2a latex agglutination test (OXOID; DR0900) according to the manufacturer's protocol. ${ }^{11}$ Antibiotic susceptibility was determined according to Clinical Laboratory Standard Institute (CLSI-guidelines 2013), for penicillin, rifampicin, cotrimoxazole, ciprofloxacin, gentamicin, tetracycline, linezolid and fusidic acid. ${ }^{4}$ Inducible clindamycin resistance was identified by 'D-zone' tests with erythromycin $(15 \mu \mathrm{g})$ and clindamycin $(2 \mu \mathrm{g})$ disks. 
Inducible and constitutive Macrolide-lincosamide-streptogramin B (MLSB) phenotypes were assessed by CLSI guidelines 2013. ${ }^{11}$ Double-Disk diffusion testing method as described by Swenson et al, 2010 was used to detect high-level and lowlevel susceptibility with mupirocin $200 \mu \mathrm{g}$ and $5 \mu \mathrm{g}$ disk. ${ }^{12}$ Glycopeptide susceptibility of MRSA was tested with vancomycin and teicoplanin (EzyMIC, Himedia) strips according to manufacturer's guidelines. Interpretations of susceptibility were done using CLSI 2013 guidelines. ${ }^{11}$ A multi-drug resistant (MDR) isolate was defined as non-susceptibility to more than 3 antimicrobial classes and a pan drug resistant (PDR) isolate was defined as non-susceptibility to all antimicrobial agents. ${ }^{12}$

Conventional PCR was done to detect the PVL gene as described by Lina et al. (2009) using lukS PV(5-ATCATTAGGTAAAATGTCTGGACATGATCC A-3) and lukFPV(5-GCATCAA STGTATTGGATAGCAAAAGC-3) as primers. ${ }^{13}$ S. aureus ATCC 25923 was used as the positive control ${ }^{14}$ while $S$. aureus ATCC 25913 was used as the negative control. ${ }^{15}$ DNA was extracted with the Wizard® Genomic DNA Purification Kit. ${ }^{16}$ After amplification for 30 cycles $\left(30\right.$ s denaturation at $95{ }^{\circ} \mathrm{C}$, 60s annealing at $55{ }^{\circ} \mathrm{C}$ and one minute extension at $72{ }^{\circ} \mathrm{C}$ ), the PCR products were resolved by electrophoresis through $2 \%$ agarose gel. This was followed by ethidium bromide staining and analysis to visualise bands at $433 \mathrm{bp}$. PCR was optimized to identify the best annealing temperature $\left(55^{\circ} \mathrm{C}\right)$ and primer concentration $(0.3 \mu \mathrm{M})$. Analytical sensitivity of the procedure was done for ten-fold serial dilutions of $S$. aureus (the positive control). Lower limit of detection was $3 \times 10^{4} \mathrm{CFU} / \mathrm{mL}$. Sensitivity was increased up to $3 \times 10^{3} \mathrm{CFU} / \mathrm{mL}$ by increasing the number of cycles to 40. All hundred samples were subjected to the optimised PCR procedure with positive and negative strains included in each run.

Descriptive analysis was employed in investigating the distributions of variables between the HA and CA groups using SPSS16. Categorical variables between the two groups were compared by means of the chi-square test or Fisher's exact test. Range was used to assess the statistical dispersion of the data set. Statistical significance was assumed if $\mathrm{P}$ value was $<0.05$.

\section{Results}

One hundred MRSA isolates were tested in the study. The mean and median ages of the CA-MRSA group were 45 years and 57 years and that for HA-MRSA were 46.67 years and 56years respectively. The basic demographics of MRSA positive patients are shown in Table 1. 
Table 1: Socio-demography of patients with CA-MRSA and HA-MRSA infections

\begin{tabular}{|c|c|c|c|c|c|c|}
\hline & \multirow{2}{*}{$\begin{array}{c}\text { CA } \\
\text { MRSA } \\
\text { count }\end{array}$} & \multirow{2}{*}{$\begin{array}{c}\text { HA } \\
\text { MRSA } \\
\text { count }\end{array}$} & \multirow[t]{2}{*}{$\begin{array}{l}\text { Odds } \\
\text { ratio }\end{array}$} & \multicolumn{2}{|c|}{$\begin{array}{c}95 \% \text { Confidence } \\
\text { Interval }\end{array}$} & \multirow[t]{2}{*}{$\begin{array}{c}\mathbf{P} \\
\text { value }\end{array}$} \\
\hline & & & & Lower & Upper & \\
\hline Female & 21 & 7 & 0.724 & 0.257 & 2.040 & 0.541 \\
\hline Male & 58 & 14 & & & & \\
\hline Sinhalese & 14 & 70 & .257 & .082 & .806 & 0.020 \\
\hline *Non- & 7 & 9 & & & & \\
\hline \multicolumn{7}{|l|}{ Sinhalese } \\
\hline$<45$ years & 9 & 34 & 993 & .375 & 2.624 & 0.988 \\
\hline$>45$ years & 12 & 45 & & & & \\
\hline
\end{tabular}

*Non-Sinhalese: Tamil, Muslim and other ethnic groups

In this study, the prevalence of HA-MRSA was 79\% (70.9\%- $87.1 \%)$ and CA-MRSA was $21 \%(12.9 \%-29.1 \%)$. The majority (92) were clinical samples and 8 were screening samples. Skin and soft tissue infections were the most common infection among all subjects. Blood and respiratory specimens had only HA-MRSA infections (Table 2).

Table 2: Type of samples

\begin{tabular}{lcccc}
\hline & \multicolumn{2}{c}{ CA-MRSA } & \multicolumn{2}{c}{ HA-MRSA } \\
\cline { 2 - 5 } & No & \% & No & \% \\
\hline Screening & 3 & 37.5 & 5 & 62.5 \\
Clinical & 18 & 19.6 & 74 & 80.4 \\
Blood stream infection & - & & 5 & 6.8 \\
Respiratory tract infection & - & & 3 & 4.1 \\
Skin/soft tissue infection/pus & 16 & 88.9 & 61 & 82.4 \\
Sterile fluid & 1 & 5.6 & 5 & 6.8 \\
Urinary tract infection & 1 & 5.6 & - & \\
\hline
\end{tabular}

Antibiotic susceptibility pattern is shown in Table 3. Resistance rates were significantly higher for fusidic acid, cotrimoxazole, tetracycline, ciprofloxacin, gentamicin, and clindamycin among isolates of HA-MRSA which showed the MDR phenotype.

All isolates were susceptible to rifampicin and linezolid but resistant to penicillin. The distribution of glycopeptide MIC values among the two groups differed. There were no glycopeptide intermediate or resistant $S$. aureus isolates. Prevalence of mupirocin resistance was $6 \%$. All resistant isolates were in the HA-MRSA group. Four isolates showed high-level resistance and two isolates showed low-level resistance.

The proportion of PVL gene among HA-MRSA isolates was 3.8\% whereas proportion of PVL among CA-MRSA isolates was $95.2 \%(\mathrm{p}<0.001)$. 
Table 3: Interpretation of antimicrobial susceptibility testing of the isolates

\begin{tabular}{|c|c|c|c|c|}
\hline Antibiotic & $\begin{array}{c}\text { Susceptibility } \\
\text { result }\end{array}$ & $\begin{array}{c}\text { HA MRSA } \\
n\end{array}$ & $\begin{array}{c}\text { CA MRSA } \\
n \\
\end{array}$ & $P$ value \\
\hline Fusidic acid $30 \mu \mathrm{g}$ & Resistant & 35 & $\mathbf{0}$ & $<0.001$ \\
\hline Cotrimoxazole & Resistant & 34 & 1 & $<0.001$ \\
\hline Tetracycline $30 \mu \mathrm{g}$ & Resistant & 56 & 1 & $<0.001$ \\
\hline Ciprofloxacin $5 \mu \mathrm{g}$ & Resistant & 61 & 1 & $<0.001$ \\
\hline Gentamicin $10 \mu \mathrm{g}$ & Resistant & 46 & 3 & $<0.001$ \\
\hline $\begin{array}{l}\text { Inducible and constitutive } \\
\text { clindamycin resistance } \\
\text { (Erythromycin } 15 \mu \mathrm{g}+\text { Clindamycin } 2 \mu \mathrm{g} \text { ) }\end{array}$ & Resistant & 62 & 9 & $<0.001$ \\
\hline Rifampicin $5 \mu \mathrm{g}$ & Resistant & $\mathbf{0}$ & $\mathbf{0}$ & NA \\
\hline Linezolid $30 \mu \mathrm{g}$ & Resistant & $\mathbf{0}$ & $\mathbf{0}$ & NA \\
\hline MDR (Multi drug resistance) & MDR & 57 & $\mathbf{0}$ & $<0.001$ \\
\hline XDR / PDR & XDR/PDR & $\mathbf{0}$ & $\mathbf{0}$ & NA \\
\hline \multirow{4}{*}{ Teicoplanin MIC } & $\begin{array}{l}\text { Range } \\
\text { MIC 50 } \\
\text { MIC90 }\end{array}$ & $\begin{array}{l}0.5-2 \mu \mathrm{g} / \mathrm{ml} \\
1 \mu \mathrm{g} / \mathrm{ml} \\
1.5 \mu \mathrm{g} / \mathrm{ml}\end{array}$ & $\begin{array}{l}0.5-2 \mu \mathrm{g} / \mathrm{ml} \\
1 \mu \mathrm{g} / \mathrm{ml} \\
1.5 \mu \mathrm{g} / \mathrm{ml}\end{array}$ & NA \\
\hline & Range & $0.25-3 \mu \mathrm{g} / \mathrm{ml}$ & $0.25-1 \mu \mathrm{g} / \mathrm{ml}$ & NA \\
\hline & MIC 50 & $0.5 \mu \mathrm{g} / \mathrm{ml}$ & $0.5 \mu \mathrm{g} / \mathrm{ml}$ & NA \\
\hline & MIC90 & $1 \mu \mathrm{g} / \mathrm{ml}$ & $0.5 \mu \mathrm{g} / \mathrm{ml}$ & \\
\hline
\end{tabular}

MDR -Multi drug resistant phenotype, PDR -Pan-drug resistance, XDR - Extreme drug resistance, MIC-Minimum inhibitory concentration, NA-Not applicable.

\section{Discussion}

The prevalence of CA-MRSA varies markedly worldwide. The NHSL is the largest tertiary care hospital in the country and caters to patients from all parts of the country. This study demonstrates that high proportions $(21 \%)$ of isolates are CA-MRSA at NHSL. CA-MRSA categorization was done according to the patient's history which is based entirely on epidemiological information. However, the boundaries between HA-MRSA and CA-MRSA are becoming blurred due to the movements of patients and infections between hospitals and the community. ${ }^{1}$

The majority of the study population had skin and soft tissue infections. HA-MRSA was common in invasive samples such as blood and lower respiratory samples showing that HA-MRSA is prone to cause more invasive disease. None of the demographic factors such as age and gender (except ethnicity) were significant associates among the two groups which may be due to the small sample size. However, evidence suggests that CA-MRSA causes infection in healthy, predominantly young hosts who have no predisposing co-morbidities and in certain groups (ethnic groups, MSM, sport teams). ${ }^{17,18,19}$ Clindamycin is used to treat serious infections caused by MRSA strains to suppress toxin production. ${ }^{4}$ Constitutive and inducible clindamycin resistance was significant among HA-MRSA isolates in our study confirming global evidence., ${ }^{2,3,15,17}$ CA-MRSA isolates in NHSL were significantly more susceptible to other antibiotics such as fusidic acid, cotrimoxazole, tetracycline, ciprofloxacin and gentamicin. The MDR phenotype was significantly higher in the HA-MRSA isolates than the CA-MRSA isolates. This highlights the importance of enforcing rational use of antimicrobials in the hospital setting. 
The dissemination of MRSA has led to a tremendous increase in the use of glycopeptides worldwide. All our isolates were within the susceptible range of glycopeptide MIC values, similar to other local studies. ${ }^{2}$ MIC50 shows how good an antimicrobial works intrinsically against a species while MIC90 reflects different resistance mechanisms. The vancomycin MIC's of the isolates suggest a drift towards antibiotic resistance. Teicoplanin MIC50, MIC90 and the range among CA-MRSA were less than that of HA-MRSA, reflecting the infrequent use of teicoplanin in the community setting. However, these data should be confirmed by large inter centre studies. There were five isolates which had vancomycin MIC of $2 \mu \mathrm{g} / \mathrm{ml}$ and seven isolates had teicoplanin MIC value $\geq 1.5 \mu \mathrm{g} / \mathrm{ml}$. We did not assess the clinical outcomes, hVISA strains (MIC $0.5-2 \mu \mathrm{g} / \mathrm{ml}$ ) and vancomycin creep in our study. There are reports of poor clinical outcome and increased mortality in S. aureus infection with MIC's at the upper end of the susceptible range. ${ }^{20,21}$

All isolates in our study showed susceptibility to linezolid and rifampicin, similar to other local studies. ${ }^{2,3}$ Mupirocin is a topical agent which is used to treat skin infections and to eliminate nasal carriage of $S$. aureus. Usually high-level resistance is identified as an 'independent predictor' of decolonization failure while low-level resistant strains can recolonize very commonly. ${ }^{22}$ In our study, prevalence of mupirocin-resistance was $6 \%$ which highlights the importance of ensuring restrictive use of mupirocin to prevent widespread resistance. Although double disk diffusion method has good sensitivity and specificity compared to broth dilution MIC, false negatives may occur rarely when there is a frame shift mutation in the mupA gene or silent mupA gene on the chromosome. ${ }^{5}$

Our study demonstrated significant presence of PVL gene among CA-MRSA ( $p$ $<0.001)$. Similar findings have been found globally. ${ }^{6,17,21}$

\section{Conclusion}

This study highlights the importance of collective use of clinical, microbiological and molecular tests for accurate differentiation of CA and HA infections. Antibiotic policy should be developed separately for the two groups of MRSA infections to optimise patient management.

Acknowledgement: We gratefully acknowledge the Director and staff of the Medical Research Institute, Colombo and Department of Microbiology at the National Hospital of Sri Lanka for their immense support.

Availability of data and materials: The datasets generated and/or analysed during the current study are not publicly available presently but are available from the corresponding author on request with no restriction.

Competing interest: There is no conflict of interest existing for any of the listed authors.

Funding: This research was funded by the Medical Research Institute, Colombo under the project no. 11/2013. There were no influences made on study design or methodology of collection, analysis, and interpretation of data and in writing the manuscript. 
Ethics approval and consent to participate: The study was approved by the Ethics Review Committee, Medical Research Institute under the project no. 11/2013 and the Ethics Committee of the National Hospital of Sri Lanka under reference number AA/ETH/2013.

Informed written consent was taken from the patients before taking data for the questionnaire.

\section{References}

1. Fraimow HS, Tsigrelis C. Antimicrobial Resistance in the intensive care unit: mechanisms, epidemiology, and management of specific resistant pathogens. J Crit Care Clin 2011; 279:1:163-205 doi: 10.1016/j.ccc.2010.11.002.

2. Jayatilleke K, Bandara P. Antibiotic sensitivity pattern of Staphylococcus aureus in a tertiary care hospital of Sri Lanka. Sri Lankan Journal of Infectious Diseases 2012; 2(2):13-17. doi: http://doi.org/10.4038/sljid.v2i2.4162.

3. Patabendige CGUA, Chandrasiri NS, Karunanayake LI, et al. Antimicrobial resistance in resource-poor settings -Sri Lankan experience. Regional Health Forum 2011; 15:1. p.18-26. No doi

4. Mandell GL, Bennett JE, Dolin R. Principle and Practice of Infectious Disease.7th ed 2 United states: Churchill Livingstone Elsevier; 2010. p.355-2578.

5. Otto M. Community associated MRSA: a dangerous epidemic. Future Microbiology 2007; 2(5):457-459. doi: https://doi.org/10.2217/17460913.2.5.457

6. Nichol KA, Adam HJ, Mccracken M, et al. Comparison of community-associated and health care-associated methicillin-resistant Staphylococcus aureus in Canada: results of the CANWARD 2007-2009 study. Diagn Microbiol Infect Dis 2011; 69(3):320-5. doi: 10.1016/j.diagmicrobio.

7. Song JH, Hsueh PR, Chung DR, et al. Spread of methicillin-resistant Staphylococcus aureus between the community and the hospitals in Asian countries: an ANSORP study. J Antimicrob Chemother 2011; 66(5):10619 doi: 10.1093/jac/dkr024.

8. Minnesota Department of Health. Disease control newsletter; Community-Associated Methicillin-Resistant Staphylococcus aureus in Minnesota 2004: 32(6):61-72. No doi

9. Barrow GI, Feltham RKA: Cowen and streel's: Manual for the identification of medical bacteria. 3rd ed. United Kingdom: Cambridge University Press;1993. PMC501641

10. MackieTJ, \& McCartney JE. Practical Medical Microbiology.13th ed. United States: Churchill Livingstone Elsevier;1996. p.245-248.

11. Franklin RC, Jean BP, Jeff A, et al. Performance Standards for Antimicrobial Susceptibility Testing; Twenty-Third Informational Supplement. CLSI document M100-S23. Wayne, PA: Clinical and Laboratory Standards Institute; 2013.

12. Swenson JM, Wang B, Andrew ES et al. Multicentre study to determine disk diffusion and broth microdilution criteria for prediction of high and low-level Mupirocin resistance in Staphylococcus aureus. J Clin Microbial 2010; 48(7):24692475. doi: 10.1128/JCM.00340-10.

12. Magiorakos AP, Srinjvasa A, Carey B et al. Multidrug-resistant, extensively drugresistant and pandrug-resistant bacteria: an international expert proposal for interim standard definitions for acquired resistance. Clin Microbiol Infect 2012;18(3):268281 doi: http://dx.doi.org/10.1111/j.1469-0691.2011.03570.x

13. Lina G, Piemount Y, Gamot-Gamot F et al. Involvement of Panton-Valentine Leukocidin producing Staphylococcus aureus in primary skin infections and pneumonia Clin Infect Dis 1999; 29(5):1128-32.

doi: https://doi.org/10.1086/313461. 
14. Pérez JR, Tapia CO, Herazo CH, et al. Nasal carriage of Panton Valentine leucocidin positive methicillin resistant Staphylococcus aureus in healthy preschool children. Rev. salud pública 2011; 13(5):824-832. doi: https://doi.org/10.1590/S0124-00642011000500011.

15. McDanald RR, Antonishgn NA, Hansen T, et al. Development of a triplex real-time PCR assay for detection of Panton-Valentine Leukocidin toxin genes in clinical isolates of methicillin-resistant Staphylococcus aureus. J Clin Microbial 2005; 43(12):6147-6149. doi: 10.1128/JCM.43.12.6147-6149.2005.

16. Kumar R, Yadav BR, Dev K, et al. 2008. Protocol Online logo. A simple protocol for DNA extraction from Staphylococcus aureus:

Protocol Online.http://www.protocolonline.org/prot/Protocol/A-Simple-Protocol-forDNA-Extraction-from-Staphylococcus -Aureus-4999.html.Accessed on Oct 5, 2008.

17. Song JH, Hsueh PR, Chung DR, et al. Spread of methicillin-resistant Staphylococcus aureus between the community and the hospitals in Asian countries: an ANSORP study. J Antimicrob Chemother 2011; 66(5):1061-9. doi: 10.1093/jac/dkr024.

18. Bukharie HA. A review of community-acquired methicillin-resistant Staphylococcus aureus for primary care physicians. J Family Community Med 2010; 17(3);117-120. doi: 10.4103/1319-1683.74320

19. Otto M. MRSA virulence and spread. Cell Microbiol 2012; 14(10):1513-1521. doi: https://doi.org/10.1111/j.1462-5822.2012.01832.x

20. Deresinski S. Vancomycin hetero resistance and methicillin-resistant Staphylococcus aureus. J Infect Dis 2009; 199:605-9. https://doi.org/10.1086/596630.

21. Hiramatsu K, Cui L,Kuroda $\mathrm{M}$ et al. The emergence and evolution of methicillin resistant Staphylococcus aureus. Trends Microbiol 2001; 9:486-493. https://doi.org/10.1016/S0966-842X(01)02175-8.

22. Cookson BD. The emergence of mupirocin resistance: a challenge to infection control and antibiotic prescribing practice. J Antimicrob Chemother 1998; 41:11-18. doi: http://dx.doi.org/10.1093/jac/41.1.11 